\title{
Arsenic Exposure Inhibits Angiogenesis in Zebrafish via Downregulation of both VEGFA and VEGFR2
}

Sherry G. Clendeno ${ }^{1}$, Divya Ganapathi Sankaran ${ }^{1}$, Abbas Shirinifard ${ }^{1,2}$, Catherine W. McCollum $^{3}$, Maria Bondesson Bolin ${ }^{3}$, Jan-Åke Gustafsson ${ }^{3}$, James A. Glazier ${ }^{1}$

1. Biocomplexity Institute, Department of Physics, Indiana University, Bloomington, Indiana

2. Information Sciences, St. Jude Children's Research Hospital, Memphis, Tennessee

3. Nuclear Receptors and Cell Signaling, Department of Biology and Biochemistry, University of Houston, Houston, Texas

Zebrafish intersegmental vessel (ISV) sprouting is a common animal model for study of angiogenesis and for screening of toxins and drugs that affect angiogenesis [1,2]. Zebrafish embryos develop rapidly, with onset of gastrulation at 6 hours post fertilization (hpf), somitogenesis at $10.5 \mathrm{hpf}$ and vascular development at $14 \mathrm{hpf}$ [3]. Angioblasts migrate to the midline to form the dorsal aorta $(D A)$ and, subsequently, the posterior cardinal vein by vasculogenesis. The notochord initiates angiogenic sprouting from these primary vessels by secreting sonic hedgehog $(S H H)$, which upregulates vascular endothelial growth factor A (VEGFA) expression and secretion in adjacent myotomes. Secreted VEGFA is present in free (VEGFA121) and extracellular-matrix (ECM)-bound forms (VEGFA165). In zebrafish, only VEGFA165, promotes sprouting [4]. Endothelial cells $(E C s)$ of the DA, express VEGF receptor 2 (VEGFR2), bind VEGFA and migrate up VEGFA gradients in the ECM [5]. Migrating ECs in an angiogenic sprout assume either tip or stalk phenotypes [5]. In tip ECs, binding of VEGFA to VEGFR2 upregulates VEGFR2 and D114 expression and downregulates Notch expression [6]. Tip-cell Dll4 expression upregulates Notch expression in adjacent ECs, which then downregulates VEGFR2 and D1l4 expression, inducing the stalk phenotype. Additional factors further modulate this core regulatory mechanism [1].

Arsenic $(A s)$, an environmental toxin, inhibits angiogenic ISV sprouting in zebrafish, resulting in shorter length, irregularly oriented, or entirely missing ISVs [2,7]. Our analyses of ISV growth dynamics showed that these changes result from decreased directed migration speed and perturbation of directional path-finding [7]. In cell culture, As exposure elevates reactive oxygen species and VEGFA, disrupts cell-cell junctions and affects Notch signaling, a key regulator of VEGFR2 [8]. To determine how As inhibits angiogenic sprouting in vivo, we quantified levels of the chemo-attractant VEGFA and its receptor VEGFR2 in normal ISV sprouting and under As exposure. We then developed a mechanistic computer simulation of angiogenic ISV sprouting to assess the sufficiency of these hypothesized modes of action of As.

We immuno-labeled VEGFA165 and VEGFR2 in whole zebrafish embryos and evaluated protein expression levels using three-dimensional (3D) quantitative confocal microscopy. We counterstained surrounding tissue with lens-culinaris-agglutinin ( $L C A)$. We fixed control and As-exposed $(100 \mu \mathrm{g} / \mathrm{ml}$ and $400 \mu \mathrm{g} / \mathrm{ml})$ flt1-eGFP (flt1 is VEGFR2) embryos overnight in 4\% paraformaldehyde in PBS at 18, 19, 20, 22, 24 hpf, then blocked and permeablized overnight in $1 \%$ BSA, 5\% horse serum, $1 \%$ Triton-X-100 in PBS. We then incubated embryos overnight in anti-zebrafish VEGFA165 (R\&D Systems, Minneapolis, MN, USA) at 1:100, FITC anti-GFP (Invitrogen, Grand Island, NY, USA) at 1:100, and rhodamine conjugated LCA (Vector Labs, Burlingame, CA, USA) at 1:50, followed by overnight incubation in AF633-anti-mouse secondary antibody (Invitrogen) at 1:50, all diluted in 5\% BSA, 1\% HS, in PBS. We acquired 3D 
images using a Leica SP2 MP system equipped with a 25x $0.95 \mathrm{NA}$ objective. We measured mean VEGF staining intensity around each ISV and mean VEGFR2 staining intensity profiles along the length of each ISV, using FIJI-ImageJ software [9]. We built the computer simulation using the CompuCell3D multi-cell modelling framework [10].

In control embryos, VEGFA levels increased more than three-fold from 18 to $20 \mathrm{hpf}$, then decreased $25 \%$ from 20 to $24 \mathrm{hpf}$. Peak VEGFA expression in controls at $20 \mathrm{hpf}$ coincides with initiation of ISV sprouting. In As-treated embryos, VEGFA levels increased three-fold (400 $\mu \mathrm{g} / \mathrm{ml} \mathrm{As})$ to four-fold (100 $\mu \mathrm{g} / \mathrm{ml} \mathrm{As})$ from 18 to $24 \mathrm{hpf}$. As-treatment delayed initiation of sprouting and reduced VEGFA levels at all time-points compared to controls. We did not observe the expected higher levels of VEGFR2 expression in tip cells than in stalk cells [5]. In extending sprouts in controls from 20 to $22 \mathrm{hpf}$, ISV mean VEGFR2 expression decreased by $50 \%$. At $24 \mathrm{hpf}$, VEGFR2 expression in the tip-cell region returned to $20 \mathrm{hpf}$ levels; however, VEGFR2 expression in the stalk-cell region was two-fold higher than in the tip-cell region. VEGFR2 expression levels were lower at all time-points in As-treated embryos.

Our preliminary computer simulations showed that: 1) The VEGF gradient created by local uptake of VEGF sufficed to support ISV sprouting and extension. 2) A small decrease in VEGF levels or increase in the rate of EC-cell uptake of VEGF resulted in sprouts that did not form or that initiated but failed to extend and then collapsed. 3) Decreasing the initial speed of EC migration decreased the VEGF level at the sprout, increasing the probability of sprout failure.

In conclusion: 1) As inhibits ISV sprouting by reducing levels of both VEGFA and VEGFR2. 2) The temporal pattern of VEGFA expression in controls supports its hypothesized role as the core regulator of angiogenic ISV sprouting. 3) Spatiotemporal VEGFR2 expression patterns in tip and stalk cells in vivo differ from those in cell culture and merit further study. 4) Our multi-cell computer simulations combining observed spatiotemporal expression patterns with hypothesized EC dynamics successfully reproduce both control and perturbed in vivo ISV dynamics.

References:

[1] K Goishi and M Klagsbrun, Current Topics in Developmental Biology 62 (2004) p. 127-152.

[2] CW McCollum et al., Birth Defects Research 93 (2011) p. 67-114.

[3] W Kimmel et al., Developmental Dynamics 203 (1995) p. 253-310.

[4] H Kawamura et al.,Blood 112 (2008) p. 3638-3649.

[5] H Gerhardt et al.,Journal of Cell Biology 161 (2003) p. 1163-1177.

[6] ND Lawson et al.,Developmental Cell 3 (2002) p. 127-136.

[7] A Shirinifard et al. Developmental Dynamics (2013) in press.

[8] L Bao and H Shi, Chemical Research in Toxicology 23 (2010) p. 1726-1734.

[9] J Schindelin et al., Nature Methods 9 (2012) p. 676-682.

[10] M Swat et al., Methods in Cell Biology 110 (2012) p. 325-366.

[11] The authors acknowledge funding by EPA R-10-0049: The Texas-Indiana Virtual STAR Center, NIGMS R01 GM077138: Competitive Renewal of Development and Improvement of the Tissue Simulation Toolkit and R01 GM76692: Competitive Renewal of Multiscale Studies of Segmentation in Vertebrate Embryos, Indiana University, Emerging Technology Fund of Texas and Robert A. Welch Foundation (E-0004). 